\title{
Programmatic correlates of maternal healthcare seeking behaviors in Ethiopia
}

\author{
Ali Mehryar Karim, Wuleta Betemariam, Samuel Yalew, Hibret Alemu, Mary Carnell, Yared Mekonnen
}

\begin{abstract}
Background: Considerable improvement in maternal healthcare use has been observed since the inception of the health extension program (HEP) in Ethiopia in 2003.

Objective: This paper evaluates the influence of HEP outreach strategies on maternal healthcare use.

Method: Cross-sectional survey of 2,916 women with children 0 to 11 months from Amhara, Oromiya, Southern Nations, Nationalities and People's, and Tigray regions, obtained between December 2008 and January 2009, were analyzed using regression models to assess the impacts of HEP strategies on maternal health outcomes.

Result: The analyses found that communities (i.e., kebeles) with relatively high prevalence of model families, higher rate of household visits by health extension workers, and higher rate of household visits by voluntary community health workers were associated with improved antenatal care use, tetanus toxoid vaccination coverage, and receiving postnatal care visits; but the strategies were not associated with deliveries attended by health professionals.

Conclusion: Although the impacts of HEP strategies on maternal healthcare use were statistically significant, they were not optimum to reach the maternal mortality reduction targets of the government of Ethiopia. The HEP needs to review and strengthen its community based strategies in order to reach its goals. [Ethiop. J. Health Dev. 2010;24 Special Issue 1:92-99]
\end{abstract}

\section{Introduction}

The government of Ethiopia (GOE) launched the health extension program (HEP) in 2003 to improve equitable access to promotive, preventive and select curative health interventions through community or kebele ${ }^{1}$ based health services-creating the backbone to reach its health related Millennium Development Goals (MDGs) $(1,2)$. To achieve universal primary health care coverage by 2009 , the HEP is establishing a health post and deploying two government-salaried female health extension workers (HEWs) in every kebele of the country. The HEWs are trained to provide basic and essential promotive, preventive and selected curative services which include communicable disease prevention and control, family planning, maternal and child health, immunization, nutrition, adolescent reproductive health, first-aid and emergency measures, hygiene and environmental sanitation, and health education and communication. Seventy-five percent of their time the HEWs provide services through household visits and community outreach activities; train families to adopt the desirable health practices and serve as 'models' in their neighborhood; and, organize communities (e.g., Idir, Ekub, Mahber) to participate in the expansion of HEP services. In many kebeles, voluntary community health workers (vCHWs) — who are 'model' family members assist the HEWs to provide the HEP services $(1,2,3)$.

Considerable achievements of the HEP have been recorded so far. Till date, over 11,000 of the required 15,000 health posts have been constructed and 30,193

\footnotetext{
${ }^{1}$ Kebeles are the smallest administrative unit with an average population of about 5,000 .
}

HEWs deployed (4). The Last Ten Kilometers (L10K) project survey (2008/09) in 115 intervention woredas ${ }^{2}$ in Amhara, Oromiya, Southern Nations, Nationalities and People's (SNNP) and Tigray-i.e., the four most populous regions of the country-found that 92 percent kebeles had at least one HEW while 76 percent kebeles had the planned two HEWs in place; and, 69 percent kebeles had the health post physically in place (4). Representative surveys conducted in Amhara, Oromiya and SNNP regions by Essential Services for Heath in Ethiopia (ESHE) project demonstrated that access to health facilities within two hours of walking distance increased from 60 percent in 2003 to above 90 percent in 2008 (5). With the HEP expansion, the maternal healthcare utilization improved (6). Between 2005 and $2008 / 09$, in the L10K areas the contraceptive prevalence rate increased more than twofold from 15 to 32 percent; antenatal care coverage also increased by more than twofold from 26 to 54 percent; tetanus toxoid coverage among pregnant women increased from 24 to 42 percent; births assisted by health professional increased from 5 to 12 percent; while postnatal care remained unchanged. Similar findings were also observed from the ESHE study (5).

Nevertheless, the current levels of maternal health care have gaps and they are less than optimum for reducing maternal mortality to reach the MDG target committed by the GOE (6). To inform the program, this study uses the L10K survey data to assess the effectiveness of HEP outreach strategies on improving maternal healthcare utilization in Ethiopia.

\footnotetext{
${ }^{2} \mathrm{~A}$ woreda is a district, the second lowest administrative unit. Ninety-five percent of the L1OK intervention woredas are rural.
}

The Last Ten Kilometers Project, JSI Research and Training Institute, Inc., House \# 2111, Kebele 03/05; Bole subCity, Addis Ababa, PO Box: 13898, Tel: 251-116-620-066; Mobile: 251-911-510-819; Fax: 251-116-630-919; email: akarim@jsi.com 


\section{Methodology}

This study takes advantage of the natural variability in the intensity of the HEP strategies across kebeles in the L10K project areas and measures impact using "doseresponse" relationships between measures of exposure to HEP strategies and the outcomes of interest-i.e., maternal healthcare utilization are expected to be higher in kebeles with higher intensity of a HEP strategy.

\section{Data}

The L10K survey-representing Tigray region and L10K intervention woredas of Amhara, Oromia, and SNNP regions-is used for this study. The two-stage cluster survey utilized WHO 30 by 7 cluster sample strategy (7) to interview 7,490 women in reproductive age from 243 kebeles (i.e., the primary sampling units) which included 2,916 women with children 0 to 11 months that were the study population. Data collection was conducted by health professionals of the respective regional health bureaus between December 2008 and January 2009. Precoded questionnaires in Amharic, Oromifa and Tigregna languages were used for the purpose.

\section{Measurements}

Maternal healthcare utilization during the most recent pregnancy and childbirth is measured among women with children 0 to 11 months. The indicators for maternal healthcare utilization considered are: 1) antenatal care $(A N C)$ coverage: the propensity of a woman obtaining ANC at least twice during last pregnancy; 2) tetanus toxoid (TT) coverage: the propensity of a woman to receive two TT injections or a booster dose during last pregnancy; 3) delivery assisted by health professional $(D A H P)$ coverage: the propensity of a woman's last birth being assisted by a trained health professional (including the $\mathrm{HEW})$; and, 4) postnatal care $(P N C)$ coverage: the propensity of a woman receiving at least one postnatal checkup from a health professional or by the HEW.

A woman's exposure to a HEP outreach strategy is measured as a contextual variable, i.e., the intensity of the HEP strategies or attributes prevailing in the kebele. The HEP attributes were chosen based on the major focus of the program's outreach activities, mainly household visits by HEWs and vCHWs and training of model families. Accordingly, the contextual measures of the intensity of the HEP strategies are: 1) Prevalence of household visits by a HEW in the kebele: for a specific respondent under study, it is the prevalence of household visits by a HEW, during the six months preceding the survey, among the other women respondents in the kebele who have or doesn't have children 0 to 11 months. 2) Prevalence of household visits by a $\mathrm{vCHW}$ in the kebele: for a given women under study, it is the prevalence of household visits by a vCHW, during the six months preceding the survey, among the other women respondents in the kebele. And, 3) prevalence of model family households in the kebele: for a woman under study, it is the prevalence of model family households (or under training for becoming model family households) among the other women respondents in the kebele.

\section{Statistical analysis}

The threats to the validity of this study, mainly the nonrandom program placement bias and the cluster survey design effect, were minimized using appropriate statistical procedures. Program placement bias would under-estimate program effects if, for example, program managers purposely intensified HEP strategies in low performing and underserved areas which would leave the comparison groups to be from high performing areasgiving negative or no program impact even if, in reality, there was a positive impact of the program. Similarly, if relatively high program intensity is recorded in areas where other factors that influence health outcomes (e.g., education, urbanization, easy access to health facilities, and presence of other developmental activities, among others) are also higher, then this study would overestimate program effect (10).

To account for non-random program placement bias the study implements logistics regression models to adjust the differentials in maternal health behavioral outcomes between kebeles due to the influence of a list of individual (age, education, marital status, number of children ever born, religion, access to radio messages and duration of residence) and contextual (household wealth, access to water sources, access to health facilities, urban settings and survey domains) factors to get the net effects of exposure to HEP strategy. The cluster-survey design effect is minimized using Taylor series linearization of the variance estimates (11).

\section{Results}

The background characteristics of the study sample are given in Table 1. The majority of the study respondents (i.e., women with children 0 to 11 months) were between the ages 20 to 30 years; almost three-quarters of the respondents had no education; almost all were married or in union; more than half had three or less children; and two-thirds were orthodox Christians. Majority of the women had no access to radio messages; three-fourth of them were in their current residence for more than 5 years; four-fifth of them were within 30 minutes from the source of drinking water; almost all were within two hours from a health facility and were from rural areas. 
Table 1: Percentage distribution of women with children 0 to 11 months by selected background characteristics $(n=2,916)$

\begin{tabular}{|c|c|c|c|}
\hline Background characteristics & $\%$ & Background characteristics & $\%$ \\
\hline Age group & & Distance to water source & \\
\hline $15-19$ & 9.2 & In compound & 6.6 \\
\hline $20-24$ & 26.8 & $<30$ min. & 73.6 \\
\hline $25-29$ & 30.5 & $30+\min$ & 19.8 \\
\hline $30-34$ & 19.1 & Distance to the nearest health facility & \\
\hline $35-39$ & 10.3 & $<30 \mathrm{~min}$ & 51.5 \\
\hline $40-44$ & 3.2 & $30 \mathrm{~min}$. to $<1 \mathrm{hr}$ & 25.4 \\
\hline $45-49$ & 1.0 & 1 to $<2$ hrs & 14.7 \\
\hline Education & & $2+\mathrm{hrs}$ & 8.4 \\
\hline Cannot read or write & 72.4 & Wealth quintile & \\
\hline Primary school & 15.9 & Lowest & 20.0 \\
\hline Higher & 11.8 & Second & 19.9 \\
\hline Marital status & & Middle & 20.8 \\
\hline Not married & 5.3 & Fourth & 20.3 \\
\hline Married/currently in union & 94.7 & Highest & 19.0 \\
\hline Children ever born & & Settings & \\
\hline 1 & 21.6 & Rural & 88.5 \\
\hline 2 & 18.7 & Urban & 11.5 \\
\hline 3 & 15.8 & Survey domain & \\
\hline $4+$ & 44.0 & Tigray & 38.3 \\
\hline Religion & & Non-L10K \& non-IFHP & 9.5 \\
\hline Orthodox & 65.8 & L10K \& non-IFHP & 6.2 \\
\hline Protestant & 12.6 & Non-L10K \& IFHP & 6.6 \\
\hline Muslim & 20.1 & L10K \& IFHP & 16.1 \\
\hline Other & 1.5 & Amhara & 20.6 \\
\hline Listens to radio & & Oromiya & 20.6 \\
\hline Almost every day & 23.1 & SNNPR & 20.6 \\
\hline At least once a week & 14.4 & & \\
\hline Less than once a week & 4.8 & & \\
\hline Not at all & 57.6 & & \\
\hline \multicolumn{4}{|l|}{ Years at current residence } \\
\hline $0-1 \mathrm{yr}$ & 5.1 & & \\
\hline $2-5$ yrs & 19.1 & & \\
\hline $6-10$ yrs & 16.1 & & \\
\hline$>10$ yrs & 59.7 & & \\
\hline
\end{tabular}

Table 2 describes the HEP intensity measures, the current levels of maternal health seeking behaviors, and the unadjusted bivariate relationships between the measures of exposure to HEP strategies and the maternal healthcare utilization indicators. On average, 34 percent of the women were visited by a HEW during the six months preceding the survey. For the purpose of the unadjusted analysis the respondents were ranked according to prevalence of household visits by a HEW in the kebele and then divided into quintiles from one (lowest) to five (highest). Accordingly, the prevalence of household visits by a HEW in the kebele was 5 percent in the lowest program intensity quintile and 67 percent in the highest intensity quintile. Similarly, the respondents were again grouped into five program intensity quintiles based on the prevalence of household visits by a vCHW in the kebele and again according to the prevalence of model families in the kebele.

Maternal healthcare utilizations among the study population were 54 percent, 60 percent, 16 percent and 10 percent for ANC, TT, DAHP and PNC coverage, respectively. Whether the maternal healthcare utilization varied according to program intensity measures were assessed using Pearson's chi-square statistics. The statistically significant $(\mathrm{p}<.05)$ relationships were in the expected directions i.e., relative high maternal healthcare utilization was observed with relatively high program intensity between vCHW visits and ANC, TT, DAHP and PNC coverage; and between presence of model families and TT and PNC coverage. However, spurious relationships, but statistically significant $(\mathrm{p}<.05)$, were observed between ANC coverage and prevalence of household visits by a HEW; between DAHP coverage and all the three measures of program exposure; and between presence of model families and ANC coverage. Nevertheless, the observed variations in the maternal health seeking behaviors between program intensity exposure categories in unadjusted bivariate analyses may be biased due to variations in some other factors between the exposure groups that influence maternal health seeking behaviors (for example, education, access to services, urbanization, among others). Accordingly, logistics regression models were implemented afterwards to get the unbiased (adjusted) program effects. 
Table 2: The study population ranked in quintiles from lowest to highest according to the intensity of the three HEP intensity measures; and ANC, TT, DAHP and PNC coverage within the quintiles

\begin{tabular}{|c|c|c|c|c|c|c|}
\hline Kebele level HEP intensity measures & & $\begin{array}{l}\text { ANC } \\
\text { coverage }\end{array}$ & $\begin{array}{l}\text { TT } \\
\text { coverage }\end{array}$ & $\begin{array}{l}\text { DAHP } \\
\text { coverage }\end{array}$ & $\begin{array}{l}\text { PNC } \\
\text { coverage }\end{array}$ & $\begin{array}{l}\text { No. of } \\
\text { women }\end{array}$ \\
\hline \multicolumn{7}{|l|}{$\begin{array}{l}\text { Prevalence of } \mathrm{HH} \text { visits by a } \mathrm{HEW} \\
\text { quintile }\end{array}$} \\
\hline Lowest & 5.2 & $61.1^{\star \star \star}$ & $59.0^{*}$ & $59.0^{\star \star \star}$ & 8.8 & 588 \\
\hline Low & 20.4 & 54.0 & 56.9 & 56.9 & 9.1 & 336 \\
\hline Middle & 32.9 & 53.0 & 58.9 & 58.9 & 8.5 & 552 \\
\hline High & 46.5 & 51.9 & 64.8 & 64.8 & 9.7 & 576 \\
\hline Highest & 67.2 & 48.4 & 60.1 & 60.1 & 12.6 & 564 \\
\hline Total & 33.9 & 53.7 & 59.9 & 59.9 & 9.7 & 2,916 \\
\hline \multicolumn{7}{|l|}{$\begin{array}{l}\text { Prevalence of } \mathrm{HH} \text { visits by a vCHW } \\
\text { quintile }\end{array}$} \\
\hline Lowest & 0.2 & $44.1^{* \star *}$ & $52.7^{\star * \star}$ & $52.7^{\star \star *}$ & $4.3^{* * *}$ & 300 \\
\hline Low & 6.6 & 46.8 & 60.0 & 60.0 & 5.4 & 588 \\
\hline Middle & 13.9 & 53.1 & 62.0 & 62.0 & 8.7 & 540 \\
\hline High & 25.3 & 62.4 & 60.4 & 60.4 & 13.1 & 624 \\
\hline Highest & 52.2 & 62.7 & 64.7 & 64.7 & 17.2 & 564 \\
\hline Total & 19.5 & 53.7 & 59.9 & 59.9 & 9.7 & 2,916 \\
\hline \multicolumn{7}{|l|}{ Prevalence of model families quintile } \\
\hline Lowest & 0.0 & $43.8^{* * *}$ & $55.9^{* \star *}$ & $55.9^{* * *}$ & 5.3 & 912 \\
\hline Low & 3.0 & 53.7 & 57.6 & 57.6 & 9.4 & 276 \\
\hline Middle & 5.2 & 67.2 & 57.9 & 57.9 & 9.9 & 564 \\
\hline High & 11.9 & 51.2 & 63.3 & 63.3 & 12.3 & 588 \\
\hline Highest & 29.8 & 57.4 & 66.3 & 66.3 & 14.4 & 576 \\
\hline Total & 9.6 & 53.7 & 59.9 & 59.9 & 9.7 & 2,916 \\
\hline
\end{tabular}

The logistics regression models estimated to demonstrate the impact of vCHW activity in the kebele on ANC, TT, DATP and PNC coverage are given in Table 3. The program intensity contextual variable 'prevalence of vCHW visiting households in the kebele' is entered in the model as a linear term to test for the 'dose response' relationship between exposure and outcome. The models indicated that women who are in kebeles with relatively high vCHW activity are more likely to receive ANC $(\mathrm{p}<.05)$, TT injection $(\mathrm{p}<.05)$ and to have PNC visit $(p<.001)$ which is net of the influence of the women's background characteristics and kebele level other contextual factors.

The models in Table 3 also indicate that having higher education was associated with improved ANC, DAHP and PNC coverage; having access to radio messages was associated with increased ANC and DAHP coverage; living within 30 minutes of an health facility was associated with improved ANC, TT and DAHP coverage; mothers with higher socio-economic status (as measured by the variable wealth quintile ${ }^{3}$ ) were more likely to use

\footnotetext{
${ }^{3}$ The wealth quintile was measured by the method described by Filmer and Pritchett (12) and widely used by demographic health surveys because the It is an indicator of the level of
}

all the four maternal healthcare services; and, women from the urban areas were more likely to have DAHP and PNC.

Interestingly, education was not the barrier against TT coverage; having the first birth was associated with more likely to have their DAHP; compared to the Orthodox Christian respondents, the Protestants were less likely to use ANC services and receive TT injection, the Muslims were more likely to receive TT injection, and the respondents from other religion were less likely to receive TT injection; living closer to the source of drinking water was associated with more likely having DAHP but less likely to have received PNC; living in urban areas was associated with not receiving TT injection; respondents from Amhara region of the L10K areas were less likely to receive ANC compared to those from Tigray region; and respondents from nonintervention areas in Tigray were more likely to receive TT compared to those from L10K areas of Amhara and Oromiya regions.

wealth that is consistent with household expenditure and income measures (13).

Ethiop. J. Health Dev. 2010;24 Special Issue 1 
Next, similar to Table 3, logistics regression models were estimated to assess the contextual effects of HEW activity in the kebele (as measured by the prevalence of household visits by a HEW in the kebele) and the presence of model families in the kebele on utilizing

Table 3: Logistic regression models showing the impact of the prevalence of household visits by a vCHW within a kebele and respondents' background characteristics on ANC, TT, DAHP and PNC coverage, $(n=2,916)$

\begin{tabular}{|c|c|c|c|c|c|c|c|c|}
\hline \multirow{3}{*}{$\begin{array}{l}\text { Independent variable } \\
\text { Kebele level prevalence of } \\
\text { vCHW HH visits }\end{array}$} & \multicolumn{2}{|c|}{ ANC coverage } & \multicolumn{2}{|c|}{ TT coverage } & \multicolumn{2}{|c|}{$\begin{array}{l}\text { DAHP } \\
\text { coverage }\end{array}$} & \multicolumn{2}{|c|}{ PNC coverage } \\
\hline & Coef. & SE & Coef. & SE & Coef. & SE & Coef. & SE \\
\hline & 0.010 & $(0.004)^{\star *}$ & 0.008 & $(0.004)^{\star *}$ & -0.006 & $(0.004)$ & 0.015 & $(0.004)^{* * *}$ \\
\hline Age & 0.017 & $(0.010)^{*}$ & -0.002 & $(0.009)$ & 0.014 & $(0.013)$ & -0.012 & $(0.014)$ \\
\hline \multicolumn{9}{|l|}{ Education (cannot read) } \\
\hline Primary school & 0.369 & $(0.120)^{\star * *}$ & 0.114 & $(0.113)$ & 0.187 & $(0.167)$ & 0.482 & $(0.175)^{\star \star *}$ \\
\hline Higher & 0.969 & $(0.200)$ & 0.115 & $(0.149)$ & 0.894 & $(0.149)^{\star \star \star *}$ & 0.622 & $(0.232)^{\star * *}$ \\
\hline Currently married/in union & 0.040 & $(0.223)$ & 0.189 & $(0.206)$ & -0.292 & $(0.244)$ & 0.087 & $(0.232)$ \\
\hline \multicolumn{9}{|l|}{ Children ever born (1) } \\
\hline 2 & 0.069 & $(0.152)$ & 0.188 & $(0.128)$ & -0.656 & $(0.183)^{\star * \star}$ & -0.066 & $(0.213)$ \\
\hline 3 & -0.034 & $(0.164)$ & 0.133 & $(0.137)$ & -0.781 & $(0.216)^{\star \star \star}$ & 0.396 & $(0.230)^{*}$ \\
\hline $4+$ & -0.147 & $(0.173)$ & 0.033 & $(0.141)$ & -0.911 & $(0.216)^{\star \star \star}$ & 0.321 & $(0.227)$ \\
\hline \multicolumn{9}{|l|}{ Religion (Orthodox) } \\
\hline Protestant & -0.486 & $(0.216)^{\star \star}$ & -0.394 & $(0.187)^{\star *}$ & -0.210 & $(0.254)$ & -0.222 & $(0.307)$ \\
\hline Muslim & 0.184 & $(0.199)$ & 0.357 & $(0.171)^{* \star}$ & -0.226 & $(0.234)$ & -0.107 & $(0.295)$ \\
\hline Other & -0.002 & $(0.367)$ & -0.610 & $(0.293)^{\star \star}$ & 0.552 & $(0.504)$ & 0.156 & $(0.522)$ \\
\hline \multicolumn{9}{|l|}{ Listens to radio (every day) } \\
\hline At least once a week & -0.202 & $(0.150)$ & -0.034 & $(0.140)$ & -0.105 & $(0.169)$ & 0.033 & $(0.213)$ \\
\hline Less than once a week & -0.100 & $(0.220)$ & -0.222 & $(0.188)$ & 0.148 & $(0.277)$ & -0.104 & $(0.313)$ \\
\hline Not at all & -0.482 & $(0.113)^{* *}$ & -0.163 & $(0.109)$ & -0.321 & $(0.144)^{\star \star}$ & -0.360 & $(0.202)^{*}$ \\
\hline \multicolumn{9}{|l|}{$\begin{array}{l}\text { Years at current residence } \\
(0-1 \mathrm{yr})\end{array}$} \\
\hline $2-5$ yrs & -0.301 & $(0.237)$ & -0.320 & $(0.219)$ & -0.107 & $(0.243)$ & 0.089 & $(0.320)$ \\
\hline $6-10$ yrs & -0.138 & $(0.246)$ & -0.234 & $(0.219)$ & 0.032 & $(0.265)$ & -0.314 & $(0.370)$ \\
\hline$>10 \mathrm{yrs}$ & -0.338 & $(0.234)$ & -0.319 & $(0.210)$ & -0.070 & $(0.229)$ & -0.052 & $(0.314)$ \\
\hline \multicolumn{9}{|l|}{$\begin{array}{l}\text { Distance to water source (in } \\
\text { compound) }\end{array}$} \\
\hline$<30 \mathrm{~min}$ & -0.536 & $(0.292)^{*}$ & -0.157 & $(0.190)$ & -0.611 & $(0.248)^{\star \star}$ & 0.755 & $(0.329)^{\star \star}$ \\
\hline $30+\min$ & -0.505 & $(0.309)$ & -0.021 & $(0.210)$ & -0.701 & $(0.274)^{\star \star \star}$ & 0.794 & $(0.364)^{\star *}$ \\
\hline $\begin{array}{l}\text { Distance to health facility } \\
(<30 \text { min. })\end{array}$ & & & & & & & & \\
\hline $30 \mathrm{~min}$. to $<1 \mathrm{hr}$ & 0.109 & $(0.120)$ & -0.054 & $(0.106)$ & -0.098 & $(0.138)$ & -0.042 & $(0.163)$ \\
\hline 1 to $<2$ hrs & -0.126 & $(0.143)$ & -0.133 & $(0.134)$ & -0.614 & $(0.220)^{\star \star \star}$ & 0.034 & $(0.213)$ \\
\hline $2+\mathrm{hrs}$ & -0.454 & $(0.208)^{\star *}$ & -0.441 & $(0.172)^{* *}$ & -0.264 & $(0.225)$ & 0.214 & $(0.258)$ \\
\hline \multicolumn{9}{|l|}{ Wealth quintiles (lowest) } \\
\hline Second & 0.320 & $(0.130)^{\star \star \star}$ & 0.081 & $(0.133)$ & 0.359 & $(0.201)^{*}$ & 0.103 & $(0.224)$ \\
\hline Middle & 0.386 & $(0.151)^{* * *}$ & 0.216 & $(0.133)$ & 0.209 & $(0.225)$ & 0.146 & $(0.229)$ \\
\hline Fourth & 0.738 & $(0.180)$ & 0.424 & $(0.158)^{\star \star \star}$ & 0.618 & $(0.239)^{* * *}$ & 0.015 & $(0.260)$ \\
\hline Highest & 0.955 & $(0.198)^{\star * \star}$ & 0.648 & $(0.182)^{\star \star *}$ & 0.820 & $(0.263)^{\star \star \star *}$ & 0.311 & $(0.282)$ \\
\hline Urban setting & -0.002 & $(0.196)$ & -0.449 & $(0.196)^{\star \star}$ & 0.496 & $(0.239)^{\star \star}$ & 0.503 & $(0.242)^{\star *}$ \\
\hline \multicolumn{9}{|l|}{$\begin{array}{l}\text { Domain (Tigray non-L10K \& } \\
\text { non-IFHP) }\end{array}$} \\
\hline Tigray (L10K \& non-IFHP) & 0.213 & $(0.287)$ & -0.383 & $(0.275)$ & 0.844 & $(0.330)^{\star \star}$ & 0.470 & $(0.324)$ \\
\hline Tigray (non-L10K \& IFHP) & 0.707 & $(0.257)^{\star \star *}$ & -0.486 & $(0.268)^{*}$ & 0.281 & $(0.303)$ & 0.383 & $(0.349)$ \\
\hline Tigray L10K \& IFHP & 0.852 & $(0.238)^{* * *}$ & -0.193 & $(0.212)$ & 0.630 & $(0.265)^{\star \star}$ & 0.347 & $(0.257)$ \\
\hline Amhara & -1.107 & $(0.218)^{* * *}$ & -0.420 & $(0.211)^{\star \star}$ & -0.371 & $(0.261)$ & -0.130 & $(0.304)$ \\
\hline Oromiya & -0.366 & $(0.269)$ & -0.552 & $(0.245)^{\star *}$ & 0.029 & $(0.305)$ & -0.392 & $(0.328)$ \\
\hline SNNPR & 0.216 & $(0.273)$ & -0.384 & $(0.223)^{*}$ & 0.136 & $(0.304)$ & -0.186 & $(0.321)$ \\
\hline Intercept & 0.176 & $(0.525)$ & 0.766 & $(0.458)^{*}$ & -0.983 & $(0.627)$ & -3.370 & $(0.657)^{\star *}$ \\
\hline
\end{tabular}

HEP: health extension program; ANC: antenatal care; TT: tetanus toxoid; DAHP: delivery assisted by health professional.

The reference category is given in parenthesis; Coef.=coefficient; SE $=$ standard error; ${ }^{*} p<0.1 ;{ }^{* *} p<0.05 ;{ }^{* \star *} p<0.01$ 
maternal healthcare services (analysis not shown ${ }^{4}$ ). The regression analyses indicated that household visits by HEWs increased TT $(p<.1)$ and PNC $(p<.001)$ coverage; and having more model families in the kebele increased ANC $(\mathrm{p}<.05)$, TT $(\mathrm{p}<.01)$ and PNC $(\mathrm{p}<.01)$ coverage.

First of all, the spurious relationships observed between program intensity measures and the maternal health seeking behaviors in Table 2 disappeared in the multivariate analyses.

Secondly policy simulations were conducted from the logistics regression models with significant HEP intensity effects on the four maternal healthcare seeking behaviors (see Figures 1a to 1h). According to the model showing the effect of $\mathrm{vCHW}$ visits on TT coverage (Figure 1d), the average TT coverage among the study population was 60.0 percent. However, if the effects of $\mathrm{vCHW}$ visits were removed from the population then the TT coverage would have reduced to 56.2 percent indicating that the net impact of $\mathrm{vCHW}$ visits was 3.8 percentage-points. If the policy to encourage vCHWs to assist the HEWs by conducting household visits is introduced in all kebeles and if its coverage level reaches the highest level then the TT coverage would further increase by 11.6 percentage-points to reach 71.6 percent, according to simulations from the logistic regression models. Similarly the statistically significant $(\mathrm{p}<.05$ or less) impacts of HEW visits in the kebele and prevalence of model families in the kebele maternal healthcare utilization indicators are also shown.

\section{Discussion}

Temporal ambiguity between exposure and the outcome is the major drawback for cross-sectional studies such as this one. Nevertheless, the observed correlations between program intensity measures and the maternal health seeking behaviors observed from the multivariate analysis are unlikely to be spurious. After removing the influence of age, education, marital status, children ever born, religion, access to radio messages, residential stability, distance from drinking water sources, distance from health facilities, socio-economic status, urban-rural settings and regional variations the study found that higher intensity of household visits by HEWs is associated with improved TT and PNC coverage; higher intensity of vCHW activity in the kebele, as measured by the prevalence of household visits by a $\mathrm{vCHW}$, is associated with improved ANC, TT and PNC coverage; and higher prevalence of model families in the kebele is also associated with improved ANC, TT and PNC coverage. However, the observed impacts of the HEP strategies were not independent of other programmatic factors. For example, the impact of household visits by
vCHWs on maternal healthcare seeking behaviors were not independent of other outreach services provided by the HEP or other developmental activities in the kebele that promoted maternal health.

The ANC package remains an important platform for reducing maternal mortality and improving neonatal survival $(14,15)$. The impacts of the HEP outreach strategies, mainly $\mathrm{vCHW}$ activities and establishing model families, on improving ANC coverage were significant; however, the HEW activities at the household level had no impact on the indicator. Absence of any influence of the household visits by HEWs on ANC coverage is not surprising. According to the L10K survey, the household visits by HEWs mainly focused on providing information on personal hygiene, promotion and use of latrine, and immunization (4) - which although are important HEP services, they are not enough to reach its maternal mortality reduction goals. The household visits by HEWs should also provide maternal healthcare information. It was encouraging to note that household visits by HEWs including the other HEP outreach strategies had significant role on improving TT coverage.

One of the targets of the GOE is to 32 percent skilled birth attendance by 2010 in order to reach its maternal mortality reduction goals (16). Despite the effort, this study has failed to detect the influence of HEP outreach community and household strategies on DAHP. Currently the DAPH coverage is only 16 percent which needs to be doubled by next year to reach the goal. Even though the HEWs are being trained on skilled births, there acceptance by the community has been low (6). Therefore, the HEP outreach activities need to adopt strategies to promote HEWs to attend deliveries.

PNC within 48 hours of childbirth, preferable within 24 hours, may prevent maternal deaths because most of the maternal deaths take place during that time (15). Early postnatal visits are also beneficial for improving neonatal survival (17). It was encouraging to see that the current HEP outreach strategies are associated with improved PNC coverage. However, the current level of PNC coverage is far less than optimum (only 10 percent) with more than two-thirds of the PNC visits taking place after 48 hours of childbirth (4). The HEP will need review its community outreach strategy to ensure PNC visits with 48 hours of childbirths. Alongside promoting HEW assisted deliveries and PNC the program also needs to develop referral linkages with emergency obstetric services to tackle delivery and postpartum complications that are not managed by the HEWs.

\footnotetext{
${ }^{4}$ Please contact the lead author to obtain the model estimates.
} 


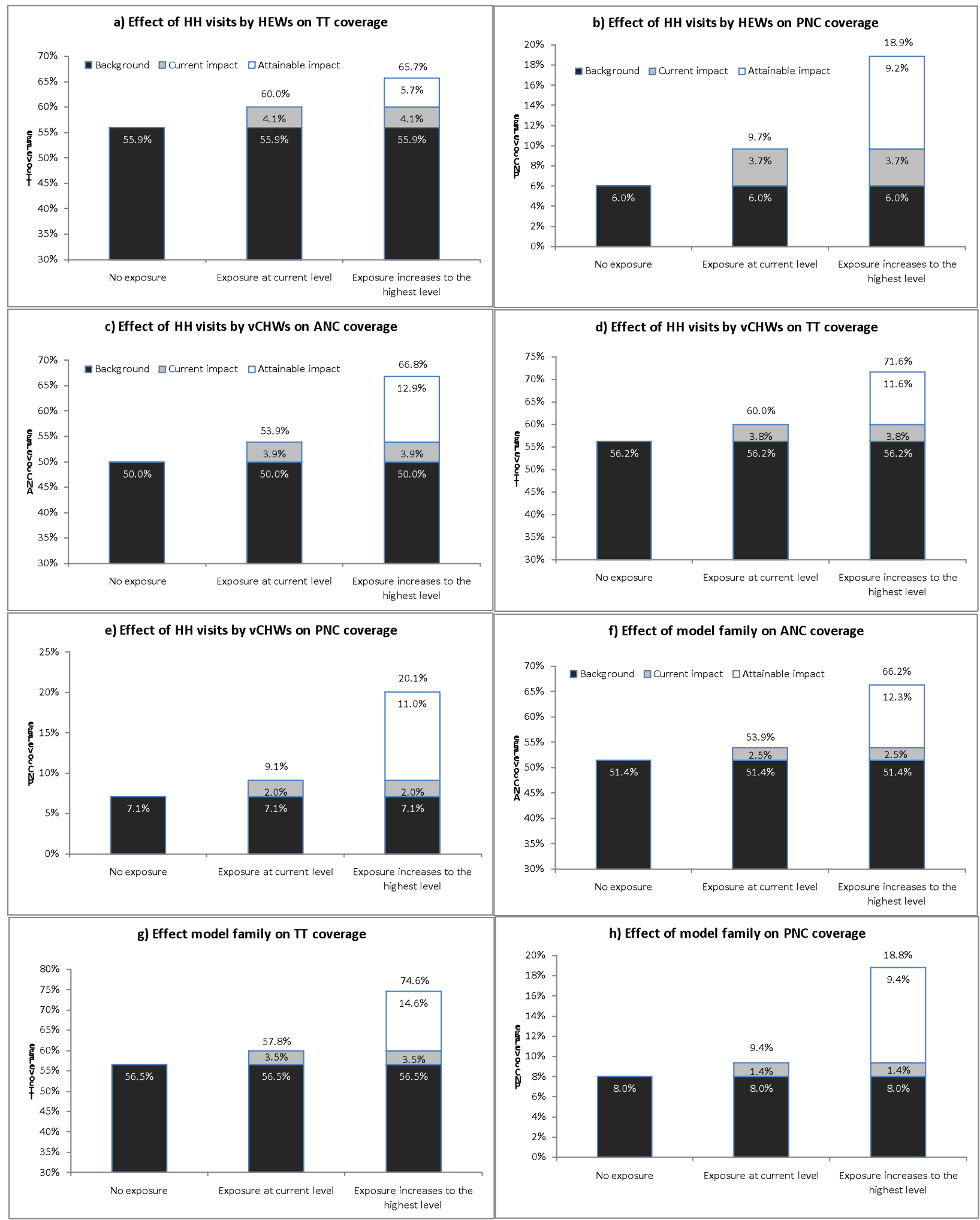

Figure 1: Simulation of the effects of HEP intensity measures on maternal health services coverage that were found statistically significant in the logistics regression models 
In conclusion, the impacts of the HEP outreach strategies on maternal health seeking behaviors were statistically significant; however, programmatically, they were less than optimum to reach the maternal health targets set by GOE. Moreover, the HEP has not yet demonstrated any impact on improving skilled birth attendance that is critical to achieve the maternal mortality reduction goals. Therefore, there is a formidable task in front of the GOE to reach its maternal mortality reduction goals.

Nevertheless, this study has demonstrated favorable aspects of the HEP upon which the future maternal healthcare programs can be built. There is evidence from this study that large scale community based primary health care program can be effective to improve health outcomes. The study shows that training model families and promoting community health workers to provide the right knowledge and skills to households can lead to improved maternal healthcare utilization. However, the mechanism by which model families in the community improves health outcome is not clear. It could be that the model families are playing a role model in the community from where other members in the community are also adopting healthy behaviors. Alternately, the higher prevalence of model family could be reflecting the areas with better performing HEWs which had lead to improved maternal healthcare utilization.

It would be important to note that the intensification of the HEP outreach strategies to increase demand for maternal healthcare services should be complemented by maintaining the quality of the healthcare services to sustain the created demand.

\section{References}

1. Temiess W. Health extension program in Ethiopia: Towards better access to health services for the rural poor. Federal Democratic Republic of Ethiopia, Ministry of Health, Quarterly Health Bulletin 2008; 1(1): 3-9

2. Girma S, Yohannes A, Kitaw Y, Ye-Ebiyo Y, Seyoum A, Desta H, et al. Human Resource Development for Health in Ethiopia: Challenges of Achieving the Millennium development Goals. Ethiopa.J.Health Dev. 2007;21(3):216-31.

3. Wakabi W. World report: Extension workers drive Ethiopia's primary health. Lancet 2008;372(9642):880.

4. The Last Ten Kilometers Project. Baseline Household Health Survey: Amhara, Oromiya, SNNP and Tigray. JSI Research \& Training, Inc. 2009; Addis Ababa, Ethiopia.
5. Essential Services for Health in Ethiopia (ESHE). Household End-line Survey Synthesis Report. Addis Ababa; 2008 September.

6. Koblinsky M, Tain F, Gaym A, Karim A, Carnell M, Tesfaye S. Responding to the challenge-The Ethiopian Health Extension Programme and back up support for maternal health care. Ethiopa.J.Health Dev. Forthcoming 2009.

7. Lemeshow S, Robinson D. Surveys to measure programme coverage and impact: A review of the methodology used by the Expanded Programme on Immunization. World Health Statistics Quarterly, 1985;38:65-75.

8. The Federal Ministry of Health. Implementation Guidelines of Health Extension Program. Addis Ababa, February 2007.

9. Hutchinson P, Wheeler J. Advanced methods for evaluating the impact of family planning communication programs: evidence from Tanzania and Nepal. Studies in Family Planning, 2006;37(3):169-186.

10. Angeles G, Guilkey D, Mroz T. Purposive program placement and the estimation of family planning program effects in Tanzania. Journal of the American Statistical Association, 1998;93(33):884899.

11. StataCorp. 2009. Stata Statistical Software: Release 11. College Station, TX: StataCorp LP.

12. Filmer D, Pritchett L. The effect of household wealth on educational attainment: Evidence from 35 countries. Population and Development Review, 1999;25(1):85-120.

13. Rutstein, S. Wealth versus expenditure: Comparison between the DHS wealth index and household expenditures in four departments of Guatemala. Calverton, Maryland: ORC Macro. 1999.

14. Carroli G, Rooney C, Villar J. How eff ective is antenatal care in preventing maternal mortality and serious morbidity? An overview of the evidence. Paediatr Perinat Epidemiol 2001;15(Suppl 1):1-42.

15. Cambell O, Graham W. Strategies for reducing maternal mortality: getting on with what works. Lancet 2006;368: 1284-1299.

16. HSDP III. Chapter 3.9 Goals, Objectives, Strategies and Key Activities of HSDP III; Annex 3: Detailed Programmatic Indicators and Targets of HSDP, 2005.

17. Darmstadt G, Bhutta Z, Cousens S, Adam T, Walker $\mathrm{N}$, Bernis L. Evidence-based, cost-effective interventions: how many newborn babies can we save? Lancet 2005;365:977-88. 